Supporting Information

\title{
Leveraging AQbD Principles for Development of Challenging Drug Substance Stability-Indicating Methods
}

Elizabeth M. Yuill*, Kevin M. Ileka, Thomas E. La Cruz, Jieming Li, Jonathan G.

Shackman, Peter I. Tattersall, and Jia Zang

Chemical Process Development

Bristol Myers Squibb

1 Squibb Dr

New Brunswick, NJ 08903

*Author to whom correspondence should be addressed.

E-mail: <elizabeth.yuill@bms.com>; Phone: <732.227.5753> 


\section{Additional Experimental Detail}

Comprehensive stationary phase/mobile phase screening. All SP/MP screens were performed at $40^{\circ} \mathrm{C}$ column temperature with a general scouting gradient. In the first tier of SP/MP screening, different MP organic modifiers (acetonitrile, methanol, and a blend of acetonitrile/methanol) were evaluated against several $\mathrm{pH}$ modifiers (i.e., TFA, ammonium acetate, and ammonium hydroxide) across acidic, neutral, and basic conditions, respectively (Figure S1). Building upon results from the first tier of comprehensive SP/MP screening, and with a smaller list of tracked impurities, the abovementioned organic modifiers were evaluated against strong and weak acid modifiers, TFA and acetic acid, in the second screening tier. Finally, only a focused list of the most challenging impurities (impurity $D$, key to the critical pair and impurity $B$, due to poor peak shape) and API were evaluated in the third tier of screens through use of acetonitrile and methanol organic modifiers with two strong acid modifiers, TFA and $\mathrm{HClO}_{4}$

Stationary phases included in the comprehensive screens spanned a broad range of manufacturers, solid supports, and ligands. The 30 columns included alkyl (with and without polar-embedded group, i.e., amide or carbamate, and with and without positive surface charge), pentafluorophenylalkyl, propylfluorophenyl, phenyl, phenylalkyl, and phenyl-hexyl chemistries, with various amounts of endcapping and on superficially porous and fully porous particles.

A Shimadzu 20 Series HPLC/photodiode array (PDA) with Binary High Pressure Gradient on Process-VP/Class-VP software was used for comprehensive SP/MP screening. 


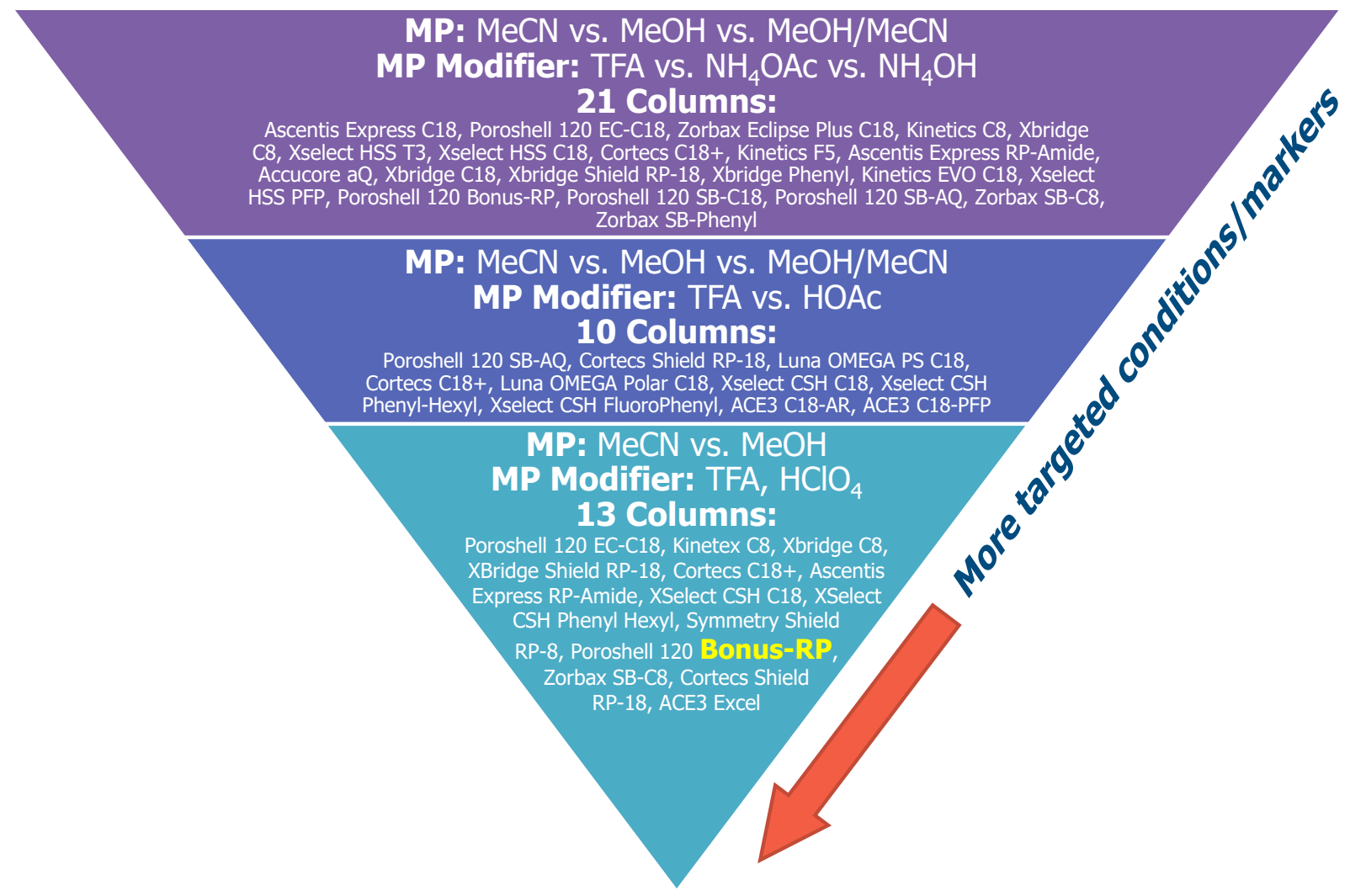

Figure S1. Detailed stationary phase/mobile phase (SP/MP) screening conditions. Comprehensive SP/MP screens were performed in three tiered stages, to build an understanding of the selectivity levers available for this complex mixture of starting material, process impurities, and degradants. The tier one screen encompassed acidic, basic, and neutral mobile phase modifiers (top segment). The tier two screening conditions were narrowed to focus only on acidic conditions (middle segment), while the final, third tier had a more targeted set of markers and only strong acid/ion pairing MP modifiers (bottom segment). Optimized SIM conditions came from the Bonus-RP hit of the third-tier screen. 


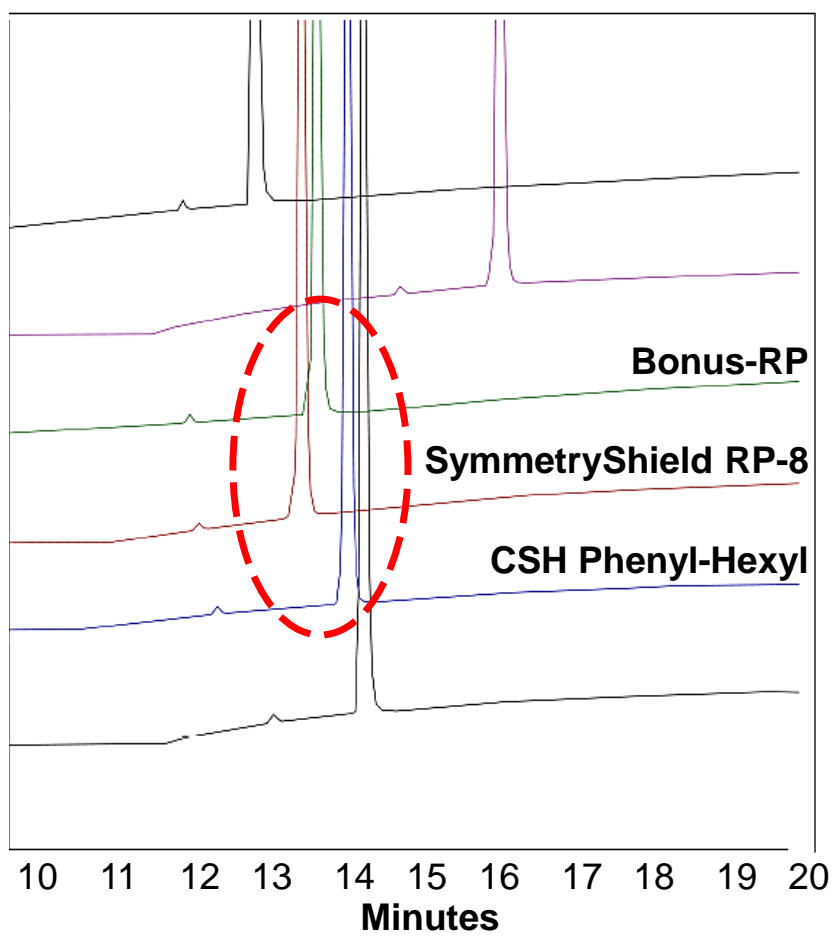

Figure S2. Selected third-tier SP/MP screening results. Subtle fronting of the API peak is shown for Bonus-RP, SymmetryShield RP-8, and CSH Phenyl-Hexyl columns (dotted red circle).

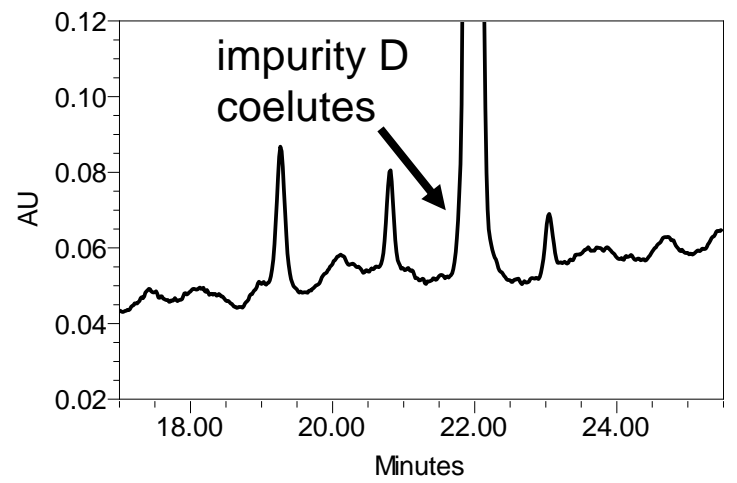

Figure S3. Selectivity for TFA pH modifier in a water/acetonitrile mobile phase system for initial tracked components. Arrow points to coelution of impurity D with the API. 

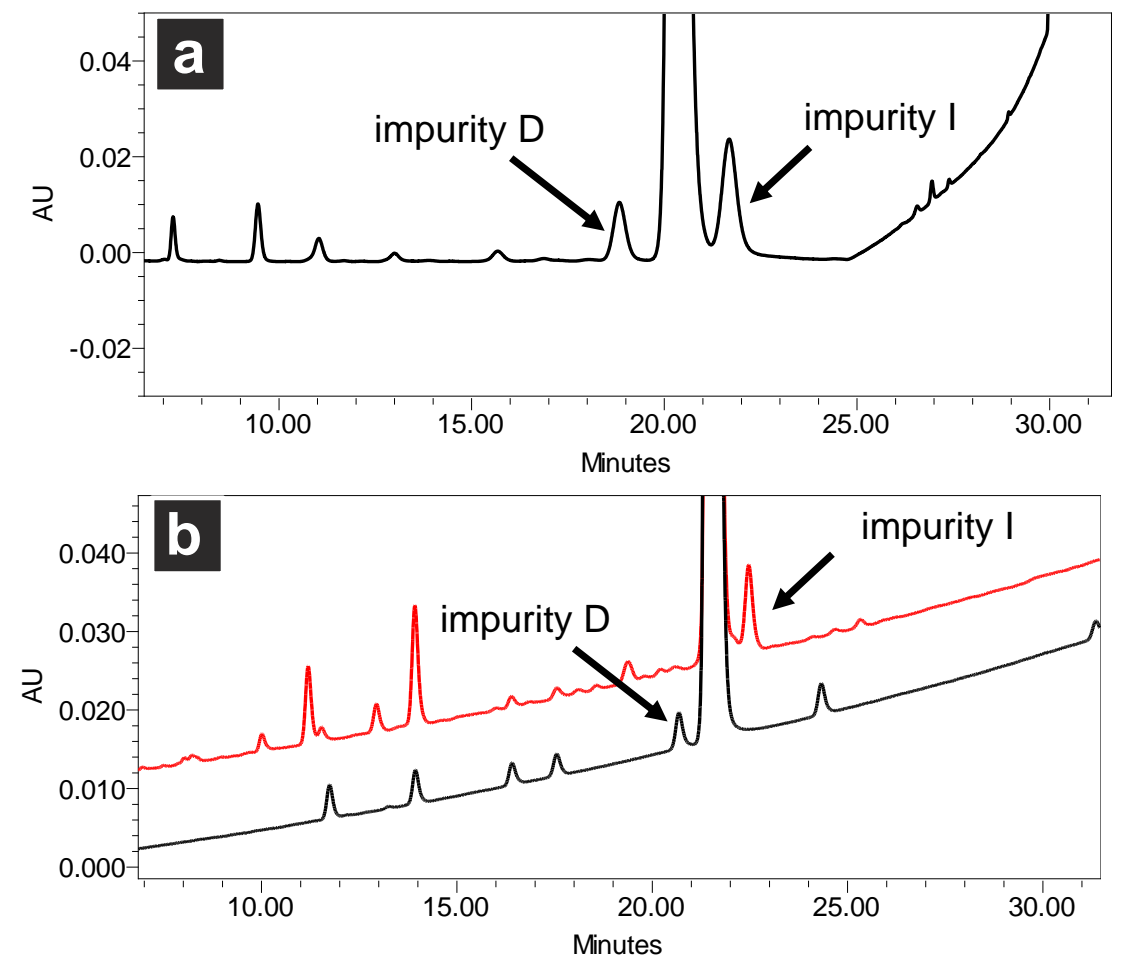

Figure S4. Balance between impurity D, API, and impurity I separation under a) isocratic and $b$ ) optimized $t G /$ column temperature conditions, both with original system (conventional HPLC), column (Agilent Zorbax Bonus-RP, $4.6 \mathrm{~mm} \times 150 \mathrm{~mm}, 3.5 \mu \mathrm{m}$ particle size), and mobile phase $\left(0.25 \% \mathrm{HClO}_{4}\right.$ in water (MP A) or in methanol (MP B)). 


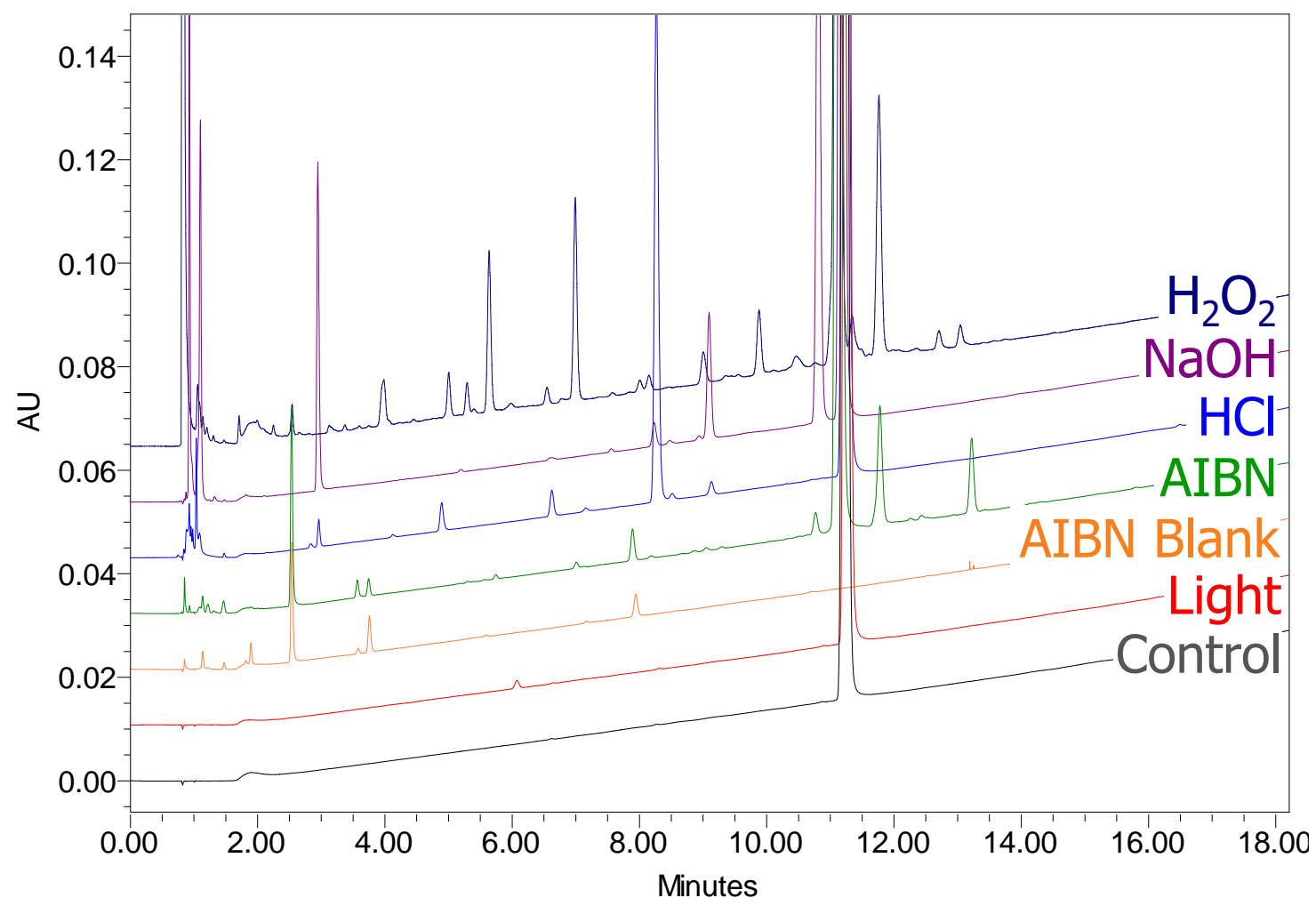

Figure S5. Overlay of final forced degradation study results on re-optimized UHPLC primary method conditions. Of note, impurity I is well separated from the API under $\mathrm{H}_{2} \mathrm{O}_{2}$ and $\mathrm{AlBN}$ conditions. 

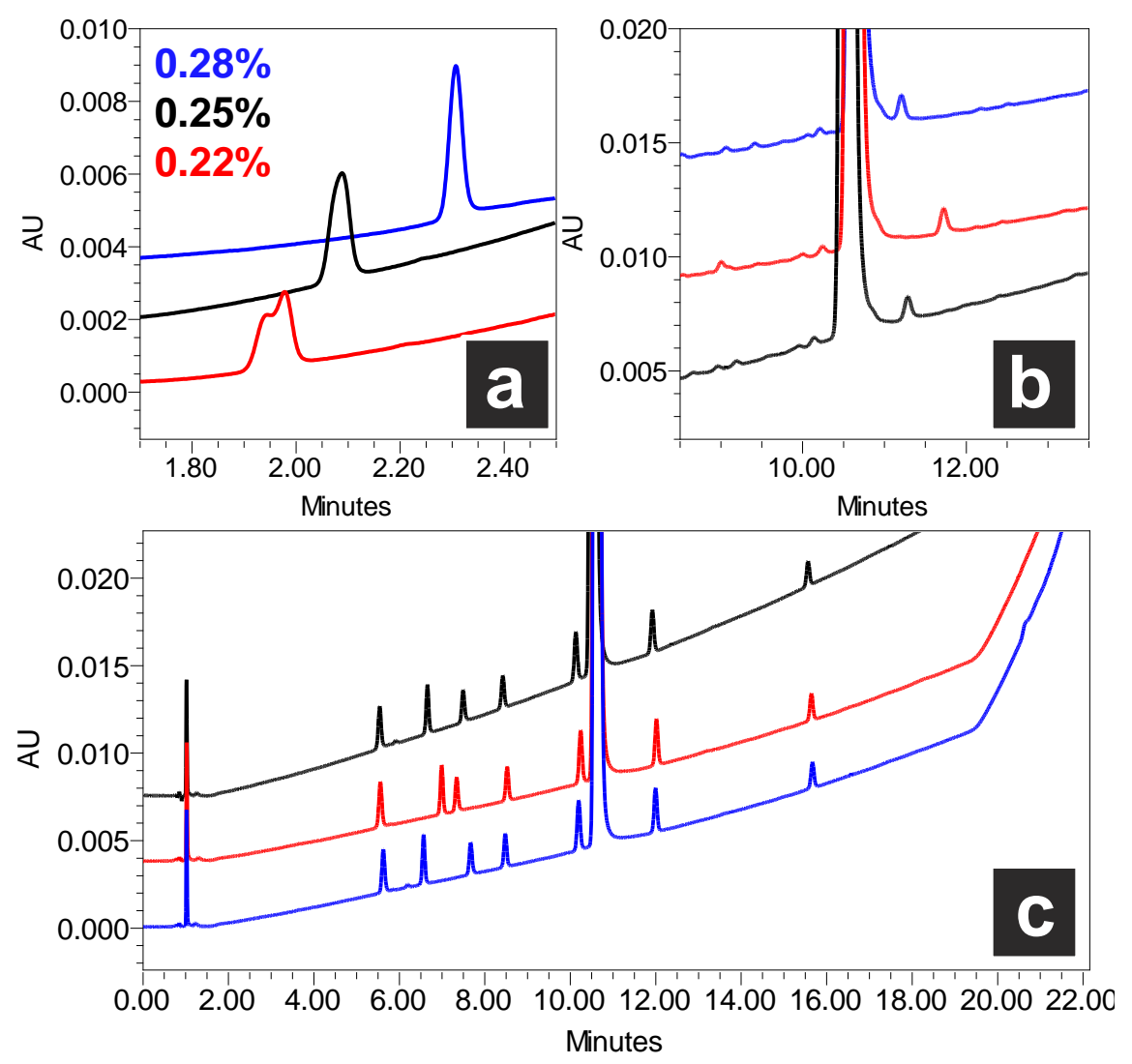

Figure S6. Tolerance of tracked components to changes in $\mathrm{HClO}_{4}$ mobile phase modifier concentration. Risk of low $\mathrm{HClO}_{4}$ mobile phase modifier concentration $(0.22 \%)$ is poor impurity G peak shape on the supplemental method (a). High $\mathrm{HClO}_{4}$ concentration $(0.28 \%)$ risks poor $\mathrm{API} / \mathrm{impurity} \mathrm{I}$ separation on the primary method (b). For these reasons, a tighter critical method parameter range of $\pm 0.02 \% \mathrm{HClO}_{4}$ was chosen. (c) Primary method showing tracked components for $0.22 \%, 0.25 \%$, and $0.28 \%$ $\mathrm{HClO}_{4}$ mobile phase modifier concentration. 


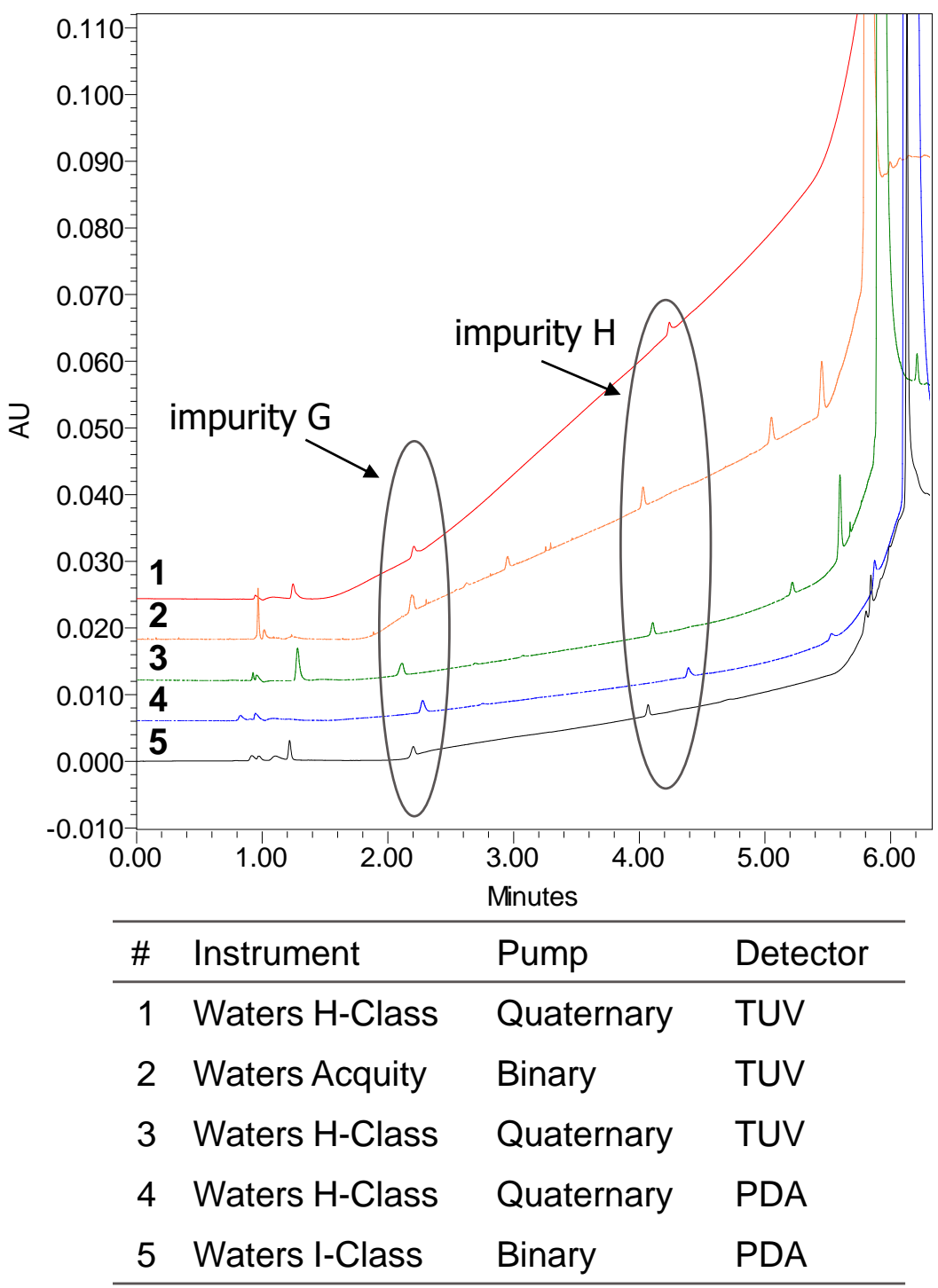

Figure S7. Robustness of UHPLC supplemental method was evaluated across different instrument types and several column lots. 

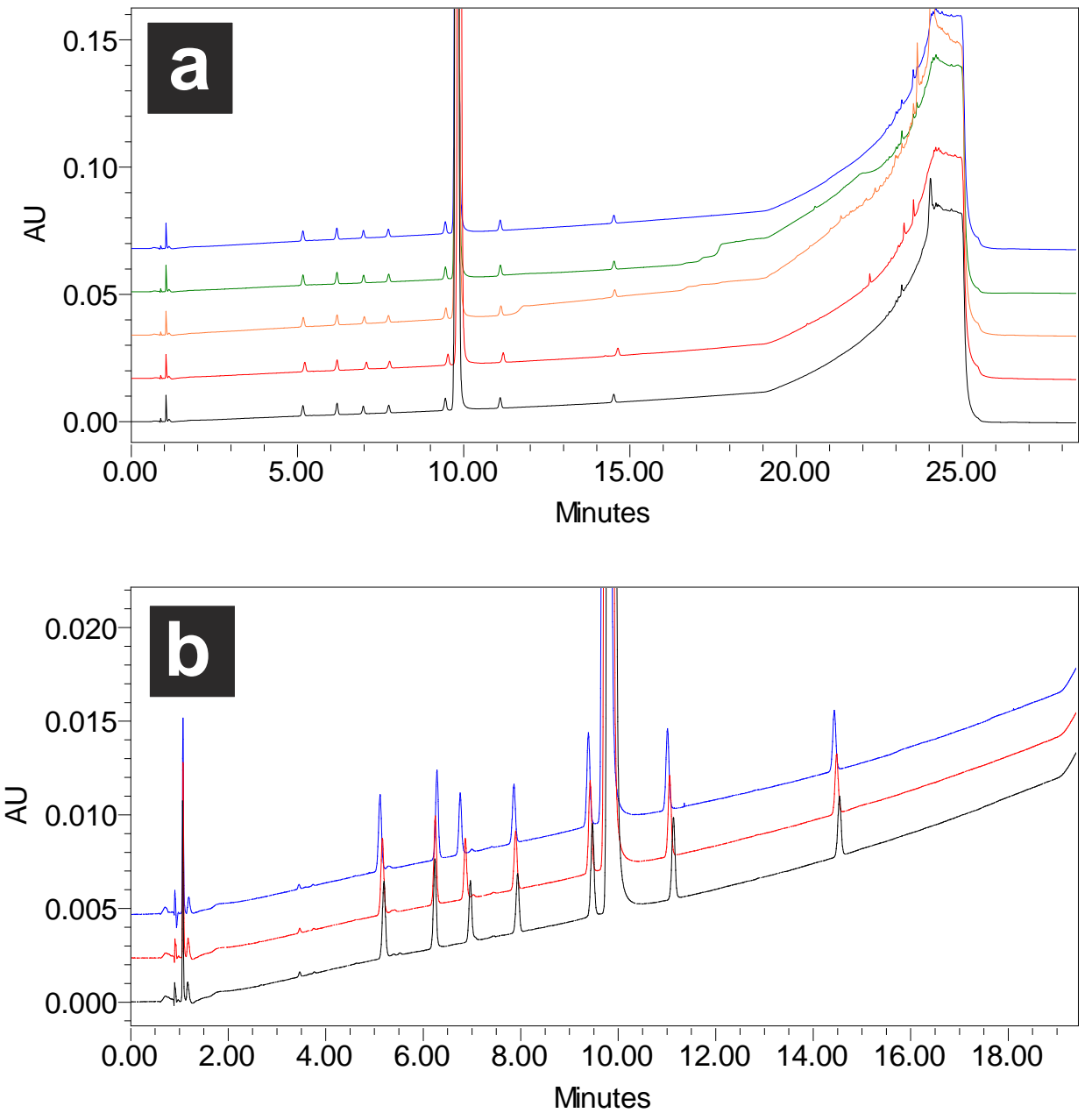

Figure S8. Mobile phase reagent selection impact on UHPLC primary method. Several different manufacturer lots of (a) methanol and (b) $\mathrm{HClO}_{4}$ were evaluated for any impact of mobile phase preparation on selectivity, peak shape, or baseline interference.

Methanol was sourced from Acros Organics, Alfa Aesar, Honeywell, Sigma-Aldrich, and VWR. 70\% $\mathrm{HClO}_{4}$ was sourced from Alfa Aesar, Avantor, and Sigma-Aldrich. 